\title{
An Efficient Macromodeling Approach for Simulating Carbon-Nanotube Field-Emission Triode Devices in Display Applications
}

\author{
Xiaojun Guo and S. R. P. Silva
}

\begin{abstract}
The advances of using carbon-nanotube (CNT) triode structure field-emission (FE) devices for display applications require an accurate and efficient SPICE-compatible device model for evaluating their electrical behaviors in the early circuit and system design stage. This letter presents a simple and efficient macromodeling approach that can accurately model the CNT triode FE devices independent of the device process and physical structures for circuit simulations.
\end{abstract}

Index Terms-Carbon nanotube (CNT), field-emission (FE) display, field-emission triode, macromodeling.

\section{INTRODUCTION}

$\mathbf{F}$ IELD-EMISSION DISPLAYS (FEDs) are characterized by superior display performances such as fast response time, wide viewing angles, wide operation temperatures, cathode-ray-tube-like colors, ultraslim features, low cost, and low-power consumption [1]. Carbon nanotubes (CNTs) have inherent advantages as a field emitter material due to their excellent field-emission (FE) characteristics, strong chemical stability, and high mechanical strength. With the advances of using CNTs as field emitters, FEDs have already shown great promise to compete in the large-size display market [2].

FEDs can be operated in either diode or triode mode devices. The triode structure with a gate electrode much closer to the cathode to extract electrons has high-emission current densities at low voltages and is therefore preferred for FEDs, which leads to lower driving voltage, high light efficiency, smooth gray scale imaging, and fast response for moving pictures [3]. In the past, much effort was expended on optimizing the process technologies and device structures to achieve higher emission current density, lower turn-on voltage, lower gate leakage for low power, and higher efficiency [1], [3]. To integrate a given FE triode device into a display system for desired performance, it is highly preferred to be able to timely and accurately evaluate the device's electrical behaviors in the early circuit and system design stage. Therefore, an efficient and accurate SPICE-compatible model must be developed for this purpose. However, the complicated device structure and FE mechanisms pose considerable challenges on device modeling. In this letter,

Manuscript received April 17, 2007; revised May 17, 2007. The review of this letter was arranged by Editor P. Yu.

The authors are with the Advanced Technology Institute, University of Surrey, GU2 7XH Surrey, U.K. (e-mail: x.guo@ surrey.ac.uk).

Color versions of one or more of the figures in this letter are available online at http://ieeexplore.iee.org.

Digital Object Identifier 10.1109/LED.2007.901389 a simple macromodeling approach is presented, which can be used to accurately model the CNT triode FE devices independent of variations of the device process and physical structures.

\section{Modeling Issues AND THE MACROMODELING APPROACH}

Due to the strong dependence of the electrical characteristics on process and physical structures, and the fairly complicated FE mechanisms of CNT, it is impractical to develop an efficient and accurate physical model for the CNT FE devices to be used in SPICE circuit simulations to evaluate their electrical performance in the early circuit and system design stage. Additionally, due to the complicated triode structure, it is also too difficult to accurately extract all the intradevice parasitic resistances and capacitances. A simple route is by using a twoterminal Fowler-Nordheim (F-N) SPICE diode model to replace the three-terminal configuration for fitting the dependence of the cathode current on the gate-cathode voltage only [4], as shown in Fig. 1(a). However, this method is not only physically inaccurate but also neglects the impact from the gate-to-cathode leakage current.

The proposed macromodeling approach is described in Fig. 1(b). Both the anode-to-cathode $\left(I_{\mathrm{ac}}\right)$ and gate-to-cathode $\left(I_{\mathrm{gc}}\right)$ currents are modeled by voltage-controlled current sources. The 1-D piecewise linear (PWL) function in SPICE is used to describe the current sources through modeling the $I_{\mathrm{ac}}-V_{\mathrm{G}-\mathrm{C}}$ and $I_{\mathrm{gc}}-V_{\mathrm{G}-\mathrm{C}}$ characteristics by directly specifying the experimentally measured data points [5]. The syntax of using the PWL function is

$$
\begin{gathered}
\overbrace{\operatorname{Gxxx}}^{(1)} \underbrace{\mathrm{n}+\mathrm{n}-}_{(2)}-\overbrace{\langle\operatorname{VCCS}\rangle}^{(3)} \underbrace{\operatorname{PWL}(1)}_{(4)} \\
\overbrace{\mathrm{in}+\mathrm{in}-}^{(5)}-\underbrace{\mathrm{x} 1, \mathrm{y} 1 \mathrm{x} 2, \mathrm{y} 2, \ldots, \mathrm{x} 100, \mathrm{y} 100}_{(6)}
\end{gathered}
$$

where

1) voltage-controlled element name;

2) positive/negative nodes of the element;

3) keyword for voltage-controlled current source;

4) PWL keyword function;

5) positive/negative controlling function;

6) experimental data points. 

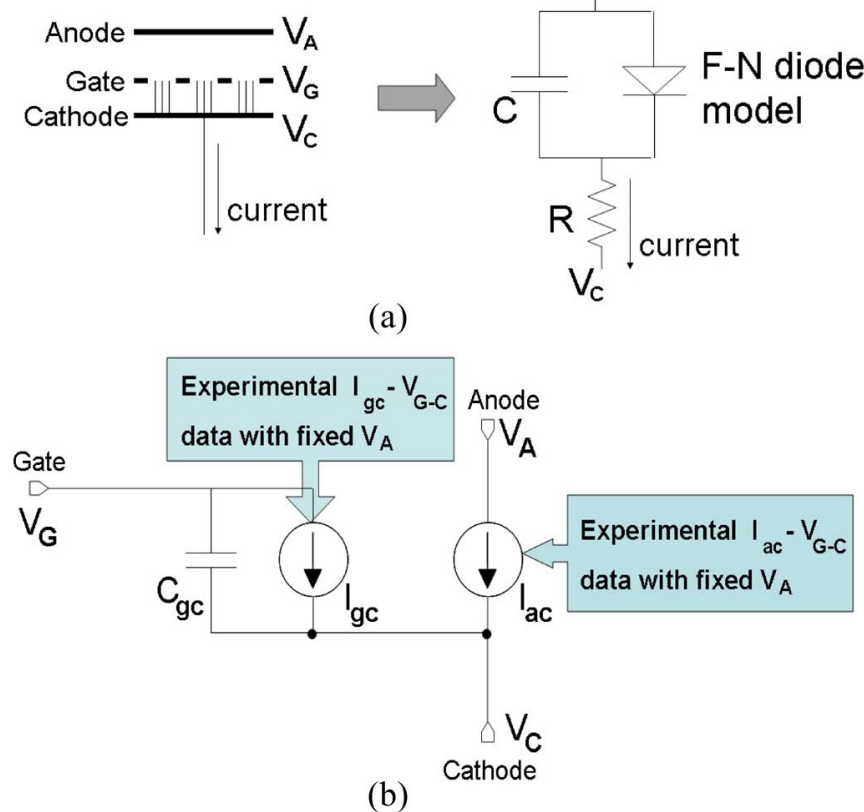

Fig. 1. (a) Simple SPICE model using F-N diode model to replace the threeterminal configuration for CNT FE triode devices. (b) Proposed macromodeling approach for developing SPICE-compatible CNT triode FE device models. The voltage-controlled current sources $I_{\mathrm{gc}}$ and $I_{\mathrm{ac}}$ are modeled by directly specifying the experimental data points via the PWL function in SPICE.

During normal electrical characterization of the FE devices, the experimental data of $I_{\mathrm{ac}}-V_{\mathrm{G}-\mathrm{C}}$ and $I_{\mathrm{gc}}-V_{\mathrm{G}-\mathrm{C}}$ characteristics at different anode voltage $\left(V_{A}\right)$ bias is obtained by varying the gate voltage $\left(V_{G}\right)$ with the fixed cathode voltage $\left(V_{C}\right)$ and anode-to-cathode voltage $\left(V_{\mathrm{A}-\mathrm{C}}\right)$. But, in an actual display matrix, the gray-scales of each FE element are modulated by changing $V_{C}$ with the fixed $V_{G}$ and $V_{A}$, and thus $V_{\mathrm{A}-\mathrm{C}}$ changes as $V_{\mathrm{G}-\mathrm{C}}$ varies for different gray-scales. However, considering the $V_{A}$ is much larger than the variations of $V_{C}$ during the full gray-scale modulation, the experimentally measured $I_{\mathrm{ac}}-V_{\mathrm{gc}}$ and $I_{\mathrm{gc}}-V_{\mathrm{gc}}$ data can still be directly used in the modeling. In Fig. 1(b), $C_{\mathrm{gc}}$ represents the parasitic capacitance between the gate and cathode, and is important for transient circuit simulations. $C_{\mathrm{gc}}$ can be extracted by using three-dimensional field solvers.

The macro-modeling approach is used to model two types of CNT FE triode devices with different structures: one is the device with CNT on silicon post structure, and the other is the device with CNTs in open aperture structure and the sidewall oxide spacer to reduce the gate leakage current. Details about the fabrication of the FE devices can be found in [6] and [7], respectively. Fig. 2 shows the perfect fitting of the simulation results based on the macro-model to the experimental data of both devices. Actually, because the experimental data of a given device is directly input into the model, the model can reproduce the real electrical characteristics of the device with no deviations, and since the modeling method doesn't need any procedures for physical equations, derivation and parameter extraction, it can also perform very efficiently by quickly developing SPICE compatible device models for the

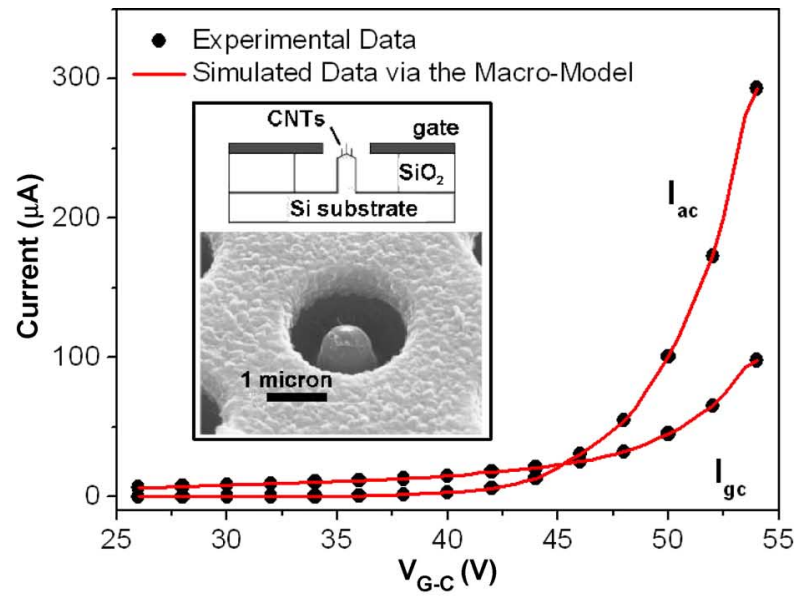

(a)

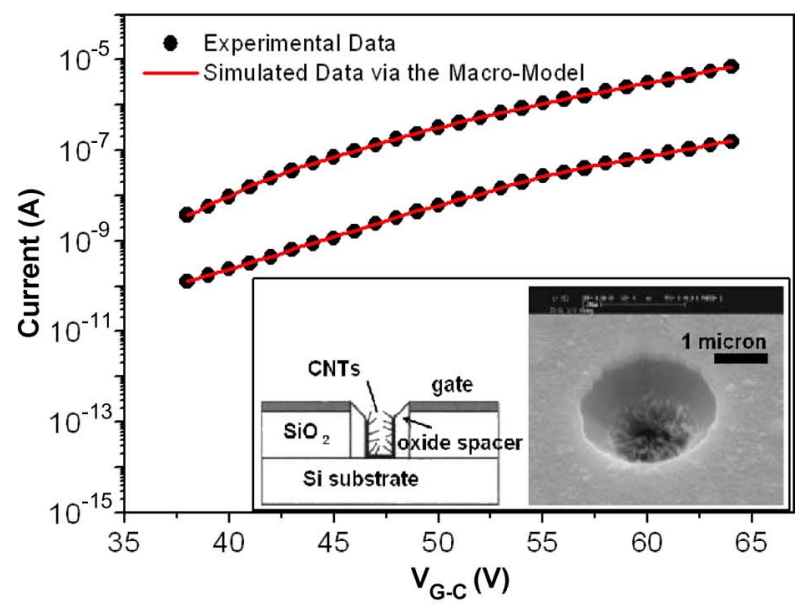

(b)

Fig. 2. Fitting of the obtained simulation results via the proposed macromodeling approach to the experimental results for two types of field emission triode devices: (a) The device with carbon nanotube on silicon post structure [6]; (b) The device with carbon nanotube in open aperture structure and the sidewall oxide spacer to reduce the gate leakage current [7].

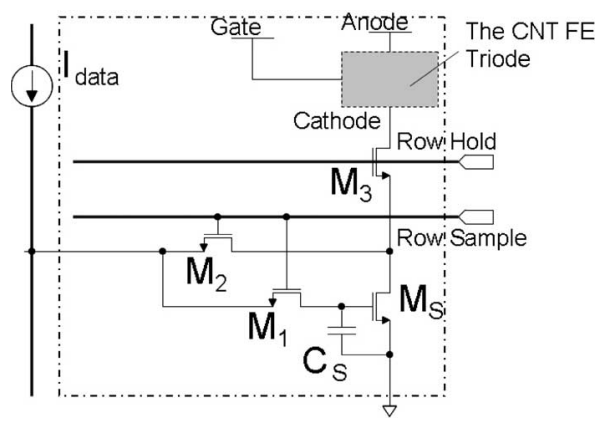

Fig. 3. Current-copier pixel driver circuit to drive and control the CNT field emission elements in active-matrix display applications.

newly built devices with novel structures or processes. The derived model is based on the input experimental data, and thus is only valid for the given CNT FE device. However, it doesn't mean that the models derived by this macro-modeling approach have less generality than those derived by a standard modeling method, since by a standard modeling method, even if a universal model can be derived, extraction of new model 


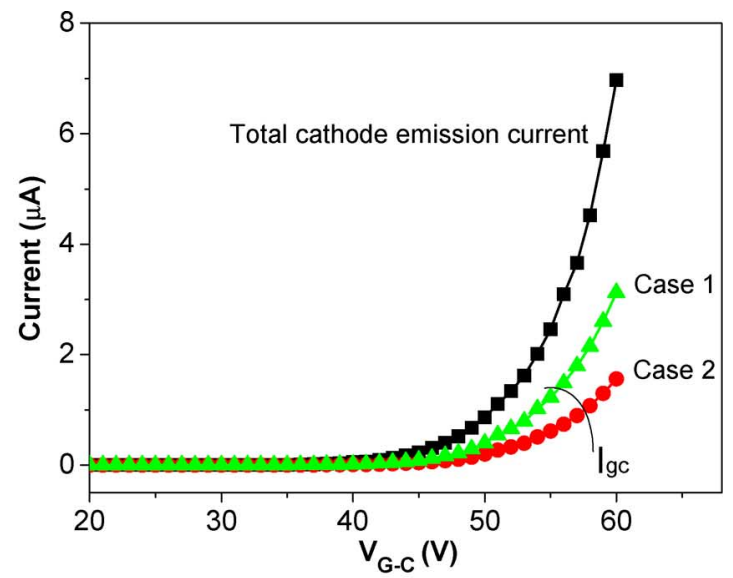

(a)

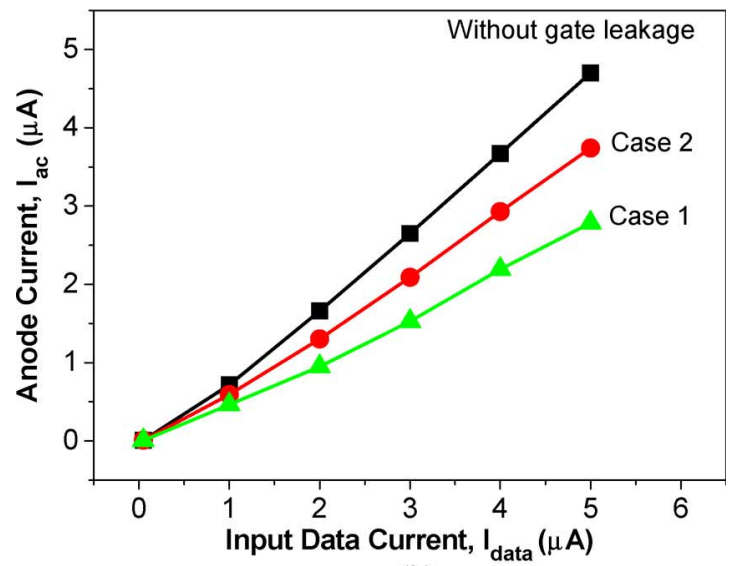

(b)

Fig. 4. (a) Electrical characteristics of the developed macro-models for the CNT FE triode devices with different gate leakage currents being used in the circuit simulation; (b) The simulated anode-to-cathode current as a function of the input data current with different gate leakage currents.

parameters is still required for a new device, which corresponds to the procedure of directly inputting the experimental data for the macro-modeling approach.

By using this macro-modeling approach, the relative complicated current-copier pixel circuit composed of four poly-Si thin-film transistors as shown in Fig. 3, which is proposed in [4] to drive and control the CNT FE triode devices to improve the FED performance, were simulated by HSPICE vended by Synopsys [5]. The details of the operation and simulation setup of the pixel circuit can be found in [4], [8]. Fig. 4(a) shows the electrical characteristics of two types of FE triode devices being used in the simulations. It is assumed, in the two cases, the total emission current from the cathode is same, but with different gate-to-cathode leakage $\left(I_{\mathrm{gc}}\right)$. Fig. 4(b) presents the simulated anode-to-cathode current $\left(I_{\mathrm{ac}}\right)$ as a function of the input data current for the pixel circuit. The increase of $I_{\mathrm{gc}}$ decreases $I_{\mathrm{ac}}$, thus result in a reduction in the brightness and also contrast. This simulation example proves the macro-modeling approach can be used to effectively model the CNT FE triode devices for circuit simulations. The results also indicate that, through considering the gate leakage current into the modeling, the simulations can quickly predict the effects of gate leakage on the display performance.

\section{CONCLUSION}

A simple and effective macro-modeling approach is introduced to quickly develop SPICE compatible device models for CNT triode FE devices in display applications. The approach has been proved to be able to accurately model the CNT triode FE devices independent of the device process and physical structures for circuit simulations.

\section{REFERENCES}

[1] S. Itoh, M. Tanaka, and T. Tonegawa, "Development of field emission displays," J. Vac. Sci. Technol. B, Microelectron. Process. Phenom., vol. 22, no. 3, pp. 1362-1366, May 2004.

[2] K. A. Dean, B. F. Coll, E. Howard, S. V. Johnson, M. R. Johnson, H. Li, D. C. Jordan, L. H. Tisinger, M. Hupp, S. G. Thomas, E. Weisbrod, S. M. Smith, S. R. Young, J. Baker, D. Weston, W. J. Dauksher, Y. Wei, and J. E. Jaskie, "Color field emission display for large area HDTV," in Proc. SID Sym. Dig., 2005, pp. 936-939.

[3] B. F. Coll, K. A. Dean, J. Jaskie, S. Johnson, and C. Hagen, "Nano-emitters for big FEDs: The carbon nanotubes solution," in Proc. Eurodisplay, 2002, pp. 219-224.

[4] X. Guo and S. R. P. Silva, "Circuit simulation of current-modulated field emission display pixel driver based on carbon nanotubes," Electron. Lett., vol. 40, no. 18, pp. 1113-1115, Sep. 2004.

[5] User's Manual, Synopsys, Mountain View, 2004.

[6] D. S. Y. Hsu, "Integrally gated carbon nanotube-on-post field emitter arrays," Appl. Phys. Lett., vol. 80, no. 1, pp. 118-120, Jan. 2002.

[7] D. S. Y. Hsu, "Microgating carbon nanotube field emitters by in situ growth inside open aperture arrays," Appl. Phys. Lett., vol. 80, no. 2, pp. 29882990, Apr. 2002.

[8] X. Guo and S. R. P. Silva, "Investigation on the current nonuniformity in current-mode TFT active-matrix display pixel circuitry," IEEE Trans. Electron Devices, vol. 52, no. 11, pp. 2379-2385, Nov. 2005. 